\title{
Molecular Clinical Biochemistry in Laboratory Medicine: The Present and the Future
}

\author{
A. R. Aroor
}

Published online: 10 May 2011

(C) Association of Clinical Biochemists of India 2011

\section{The Present}

Molecular Testing as a Significant Diagnostic Work Load in Clinical Biochemistry Laboratory

Conventional biochemistry (stat, urgent, routine and special chemistries) has made exponential progress for the last 50 years with the advent of automation and development of novel detection systems. At present it is growing in a slow phase in terms of technology and number of tests available. Moreover, the availability of dry reagent chemistry, immunochemical and nanotechnology made it possible to take the diagnostic tests nearer to the patient (point of care testing and physician office testing). However, one should not underestimate its importance in terms of utility for most common autoanalyzer based tests that are currently performed. In contrast to conventional clinical biochemistry, molecular diagnostic tests were not easily available in the initial years because of technical hurdles and biological discovery. However, the progress in automated DNA sequencing, sequencing of human genome, refinements in mass spectrometry, and improvement in polymerase chain reaction technology has revolutionized the molecular biology and genetic tests of clinical biochemistry [1]. Although, this has been evident from the work from pioneer institutions and diagnostic laboratories, smaller laboratories and many centers are either not providing many tests or not able to integrate these tests along with other tests as a molecular diagnostic service in concert

\section{A. R. Aroor $(\square)$}

Department of Medical Pharmacology and Physiology,

School of Medicine, University of Missouri,

One Hospital Drive, Columbia, MO 65212, USA

e-mail: aroora@helath.missouri.edu with molecular pathology or molecular microbiology components of central laboratory diagnostic services.

Polymerase Chain Reaction and Mass Spectrometry as Two Wings of Molecular Testing

At present, most of the clinical biochemistry laboratories providing molecular testing are done through utilization of robust polymerase chain reaction with high capacity instrumentation and the use of mass spectrometry. For example, gene polymorphism, pharmacogenomic and PCR based microbial molecular tests are offered in the biochemistry laboratory of central laboratory facility. This has been done in an integrated way by establishing a molecular diagnostic division with the other sections of clinical biochemistry laboratory. Similarly, toxicology and biochemical genetics services are offered in mass spectrometry laboratory. These developments highlight the need and provison of quality based and dedicated sections of the laboratory providing patient care without any conflicts raising the issues of overlapping tests related to microbiology, clinical biochemistry and molecular pathology.

From Simple Molecular Genetic Testing to Complex System Biology: Emergence of Molecular Laboratory Medicine

The last decade has witnessed noticeable advances in identifying biomarkers by making use of so called layered approach of molecular technologies. This approach is referred as systems biology and as the name implies, it provides an integrated layer molecular dissection of a person in terms of genomics, epigenomics, proteomics, metabolomics and bioinformatics [2, 3]. Proteomic approach may be further grouped into conventional protein 
biomarker detection, multiplex proteomics and morphoproteomics. Epigenomoics is a newly developed area of molecular diagnostics and it is emerging as an important area for exploring epigenetic markers for tumor diagnosis and monitoring. The rapid progress in systems biology has started to yield returns in terms of biomarker discovery and their utility in both academic and industrial settings.

\section{The Future}

From Molecular Diagnostics to Molecular Therapeutics: Role of Molecular Clinical Biochemistry in Clinical Translational Science and Personalized Molecular Medicine

\section{Molecular Biomarkers and Translational Science}

The contribution of clinical molecular biochemistry for clinical translational science is through use of biomarkers for diagnosis and disease monitoring. Comprehensive molecular testing in this direction requires the service of genomics (microarray, epigenomics, and DNA methylation status of specific genes) and proteomics. For the past decade, the discovery of epigenetic biomarkers has been the major development in clinical translational science. Recent research studies have demonstrated the ability of epigenetic biomarkers comprising dna methylation, epigenetic histone modifications and changes in micro RNA expression using human biological samples or small tissue samples (laser micro dissection and use of formalin fixed specimens) as an improvement over current diagnostic tools. What is more exciting in epigenomic molecular diagnostics is the recent development of quantitative single step methylation analysis with native DNA template thus enabling feasibility of epigenomic biomarker as a high throughput analysis [4]. Protein arrays are becoming valuable tools and use of biopsy specimens with small volumes offers a promising approach for the optimal evaluation of disease detection and progression. It is becoming clear that functional proteomics and nanoLCMS/MS based metabolomics is providing novel insights into molecular biochemistry of disease states $[5,6]$. The next challenge is to validate these candidate biomarkers in clinical translational science. Once the candidate markers are identified, these markers can be developed as high throughput multiplex quantitative analysis.

\section{Pharmacogenomics and Personalized Molecular Medicine}

The importance of genetic makeup an individual/s response to drug (pharmacogenetics) is being increasingly recognized and pharmacogenomic tests are being done to predict an individual's response to drugs and determine the accurate dosage [7]. In addition to pharmacogenomics, additional layers of molecular testing are pharmacoepigenomics and functional proteomics if one has to provide complete information of molecular tests for personalized molecular therapy [8-10]. These laboratory services need establishment of epigenetic and proteome laboratory as a part of molecular testing laboratory.

Fitting Molecular Diagnostic Services for Different Types of Laboratories

For small sized laboratories, initial efforts to provide tests such as gene polymorphism tests in relation to cancer risk, pharmacogenomics tests, and tests for single gene disorders and tests for microbial diagnostic services may be considered. This can easily be accomplished by setting a dedicated PCR laboratory in tertiary care hospitals affiliated to School of Medicine. In addition to PCR laboratory, some of the large sized tertiary care hospitals with well established superspecialities are capable of establishing mass spectrometry laboratory in clinical biochemistry. This will provide community based service for inherited disorders of metabolism, toxicology services and metabolomics as a part of translational research. Large sized referral laboratories in selective medical centers are in position to offer additional molecular testing services such as proteomics, transcriptomics and epigenomic tests $[11,12]$.

\section{Teaching Curriculum and Post Graduate Practical Training}

Continuing medical education program as a regional and national activity throughout the year at different centers or preconference events at regional and national meetings is the most effective means of disseminating the required expertise to establish these tests in the laboratory services of clinical biochemistry in the coming years. Hands on workshops, didactic lectures and problem based solving of few cases in the context of molecular testing is worth considering. The institutions already actively engaged in different aspects of molecular testing may be considered as centers for workshops or training. Inclusion of topics such as principles of epigenetics, human genome project, genetic variability, pharmacogenetics, phramacoepigenetics, genetic differences in drug targets and transporters, gene polymorphism of importance, different aspects of system biology may be included in the teaching program. Systems biology topics have been incorporated in many training programs in clinical biochemistry. Examples of molecular techniques for post graduate training are isolation of DNA from different tissues and samples, isolation of RNA for both gene expression and micro RNA quantitation, extraction of histones, western 
blotting in tissue specimens, PCR techniques for different molecular diagnostic tests, restriction fragment polymorphism analysis or polymorphic SNPs, gene expression studies using selective pathway based array in 96 well format, immunofluoroscence techniques and flow cytometry. Further enhancement of knowledge in these areas may be acquired through web based material and such sites may be created with the help of national societies. A comprehensive description of tests may be provided on the web site. The best way to ensure that the efforts of training are fruitful is to include molecular testing components in theoretical and practical examinations. The advanced level knowledge can be tested as a problem based case solving approach in the practical examination.

\section{References}

1. Aroor AR. Proteomics and bioinformatics in laboratory medicine: utility for diagnosis and drug design. National symposium on medical informatics and CME on hospital information system, India, 2004.
2. Shukla SD, Aroor AR. Epigenetic effects of ethanol on liver and gastrointestinal injury. World J Gastroenterol. 2006;12:5265-71.

3. Dennis YM, Wittwer CT. Molecular diagnostics: at the cutting edge of translational research. Clin Chem. 2009;55:601.

4. von Kanel T, Gerber D, Schaller A, Beamer A, Wey E, Jackson CB, et al. Quantitative one step DNA methylation analysis with native genomic DNA as a template. Clin Chem. 2011;56:1098-106.

5. Shetty TK. Metabolomics: impact on diagnosis and monitoring of inborn/acquired metabolic disorders Indian. J Clin Biochem. 2007;22:3-5.

6. Anderson NG. Adventures in clinical chemistry and proteomics A personal account. Clin Chem. 2010;56:154-60.

7. Thorn CF, Whirl-Carrillo M, Klein TE, Altman RB. Pathwaybased approach to Pharmacogenomics. Curr Pharmacogenom. 2007;5:79-86.

8. Long RM, Berg JM. What to expect from the pharmacogenomics research network. Clin Pharmacol Ther. 2011;89:339-41.

9. Rifai N. Clinical chemistry: a greater emphasis on proteomics. Clin Chem. 2010;56:682.

10. Mallinowsky K, Wolff C, Gundisch S, Berg D, Becker KF. Targeted therapies in cancer-challenges and chances offered by newly developed techniques for protein analysis in clinical tissues. J Cancer. 2011;2:26-35.

11. Lino M, Merlo A. Translating biology to the clinic: the case of glioblastoma. Curr Opin Cell Biol. 2009;21:311-6.

12. Gohar AV. Clinical applications of epigenetic markers in diagnosis and treatment of cancer. J Biological Sci. 2010;10:373-85. 